\title{
Low-noise Reconfigurable 12- to 16-bit Delta-Sigma Capacitance- to-digital Converter with Chopper Stabilization Technique
}

\author{
Byeoncheol Lee, ${ }^{1}$ Youngwoon Ko, ${ }^{1}$ Hyungseup Kim, ${ }^{1}$ Yeongjin Mun, ${ }^{1}$ \\ Seounghoon Huh, ${ }^{2}$ Dongkyu Song, ${ }^{2}$ Youngtaek Roh, ${ }^{2}$ and Hyoungho Ko ${ }^{1 *}$ \\ ${ }^{1}$ Department of Electronics Engineering, Chungnam National University, Daejeon 34134, Republic of Korea \\ ${ }^{2}$ LeoLSI Co., Ltd., 414, Hyoryeong-ro, Seocho, Seoul 06728, Republic of Korea \\ (Received April 5, 2017; accepted January 22, 2018)
}

Keywords: capacitance-to-digital converter (CDC), delta-sigma capacitance-to-digital converter, chopper stabilization, reconfigurable resolution

This paper presents a first-order delta-sigma $(\Delta \Sigma)$ capacitance-to-digital converter (CDC) with low noise characteristics and a reconfigurable resolution of 12 to 16 bits. The proposed $\Delta \Sigma$ CDC is implemented as a first-order $\Delta \Sigma$ modulator with switched capacitor (SC) integrator and comparator. The resolution can be reconfigured by the accumulator using the reconfigurable 12- to 16-bit up-counter. $\Delta \Sigma$ schemes are widely used for low-noise applications owing to the ability of the $\Delta \Sigma$ modulator to reduce in-band white noise through its inherent noise-shaping characteristic. Low-frequency colored noises such as flicker $(1 / f)$ noises still remain. In order to reduce the low-frequency colored noise component, a chopper stabilization technique is exploited using the SC integrator of the $\Delta \Sigma \mathrm{CDC}$. The proposed $\Delta \Sigma \mathrm{CDC}$ also controls the offset calibration capacitors that adjust the DC offset. This is caused by a capacitor mismatch owing to process variation and the parasitic capacitance of the input capacitive sensor. The $\Delta \Sigma \mathrm{CDC}$ is fabricated by using the standard $0.18 \mu \mathrm{m} \mathrm{1P6M}$ complementary metal-oxidesemiconductor (CMOS) process with an active area of $0.66 \mathrm{~mm}^{2}$. The total current consumption for the 16-bit $\Delta \Sigma \mathrm{CDC}$ is $141 \mu \mathrm{A}$ with a $1.8 \mathrm{~V}$ supply.

\section{Introduction}

Capacitive sensors are widely used in variable sensor applications for measuring pressure, humidity, and acceleration. Recently, many applications have begun to require sensor interface circuits of simple structure and high performance to allow enhancements such as increased resolution. Conventional capacitive sensor interface circuits consist of a capacitance-tovoltage converter and an analog-to-digital converter (ADC). ${ }^{(1)}$ These interface circuits have disadvantages in terms of area, power consumption, and complexity of the circuit. In order to achieve the above requirement, a directly conversable capacitance-to-digital converter (CDC) can be used. Several interface circuits have been studied that demonstrate a directly conversable $\mathrm{CDC}$ such as a successive approximation register (SAR) or delta-sigma $(\Delta \Sigma) \mathrm{CDC}^{(2-8)} \mathrm{SAR}$

*Corresponding author: e-mail: hhko@cnu.ac.kr http://dx.doi.org/10.18494/SAM.2018.1867 
CDCs have a high speed and low power consumption. However, they are limited in obtaining a high resolution. $\triangle \Sigma \mathrm{CDCs}$ have an advantage in obtaining a high resolution with shaped white noise. The noise-shaping characteristic of the $\Delta \Sigma$ modulator allows for the removal of white noise. Higher-order $\Delta \Sigma$ modulators can more effectively reduce the in-band white noise and allow for more precise readings of capacitance difference. However, with these enhancements, the circuit becomes increasingly complicated and requires a larger area. ${ }^{(5)}$ This paper presents a first-order $\Delta \Sigma \mathrm{CDC}$ with low noise characteristics. Improving the resolution of the first-order $\Delta \Sigma \mathrm{CDC}$ can be achieved by reducing other in-band noises.

The DC offset and low-frequency colored noise are additional degradation factors to consider. The DC offset is caused by capacitor mismatch owing to the process variation and parasitic capacitor of the input capacitive sensor. The DC offset can be removed by applying calibration capacitors in parallel with the capacitor of the sensor. ${ }^{(4,6)}$ The white noise and DC offset are thereby reduced, but low-frequency colored noises such as flicker $(1 / f)$ noise still remain. The proposed $\Delta \Sigma \mathrm{CDC}$ use the first-order $\Delta \Sigma$ modulator with switched capacitor (SC) integrator and comparator. By applying the chopper to the SC integrator, inband $1 / f$ noise to the outband is modulated. ${ }^{(9)}$ The outputs of the comparator are accumulated by the reconfigurable 12- to 16-bit accumulator. The fully integrated accumulator can reduce back-end digital processes.

\section{Operation of Proposed First-order $\Delta \Sigma \mathrm{CDC}$}

\subsection{Architecture of the proposed $\Delta \Sigma$ CDC}

The architecture of the proposed first-order $\Delta \Sigma \mathrm{CDC}$ is shown below in Fig. 1. The proposed first-order $\Delta \Sigma \mathrm{CDC}$ consists of the parasitic capacitance cancellation input stage, SC integrator, comparator, and accumulator. At the input stage of the $\Delta \Sigma \mathrm{CDC}$, the programmable capacitor array, added in parallel to the sensor capacitors, is used for DC offset control. The sensor driving voltages can be connected through internal voltage VDD and GND. The SC integrator using chopper stabilization and the comparator are operated by the first-order $\Delta \Sigma$ modulator. The outputs of the comparator are accumulated by the reconfigurable 12- to 16-bit accumulator. The internal relaxation oscillator, current reference, and IVREF generate the $1 \mathrm{MHz}$ main clock and the bias voltage used in the $\Delta \Sigma \mathrm{CDC}$.

\subsection{Operation of proposed first-order $\Delta \Sigma \mathrm{CDC}$ with chopper stabilization}

Figure 2 shows the schematic of the proposed first-order $\Delta \Sigma \mathrm{CDC}$ for the capacitive sensor. By using the SC integrator, the proposed first-order $\Delta \Sigma \mathrm{CDC}$ directly converts the capacitance difference of the capacitive sensor to digital codes. The frequencies of the nonoverlapping clocks, $\mathrm{P}_{1}$ and $\mathrm{P}_{2}$, are $512 \mathrm{kHz}$, which is half the main frequency. When switching from $\mathrm{P}_{1}$ to $\mathrm{P}_{2}$, the charges stored in the sensor and reference capacitors are delivered to the integrator. If the charge is delivered by the sensor capacitor and $C_{r e f}$ is balanced, then the output of the integrator is $V_{\text {ref. }}^{(2)}$ The polarity of the charge delivered by $C_{\text {ref }}$ is adjusted to obtain the 


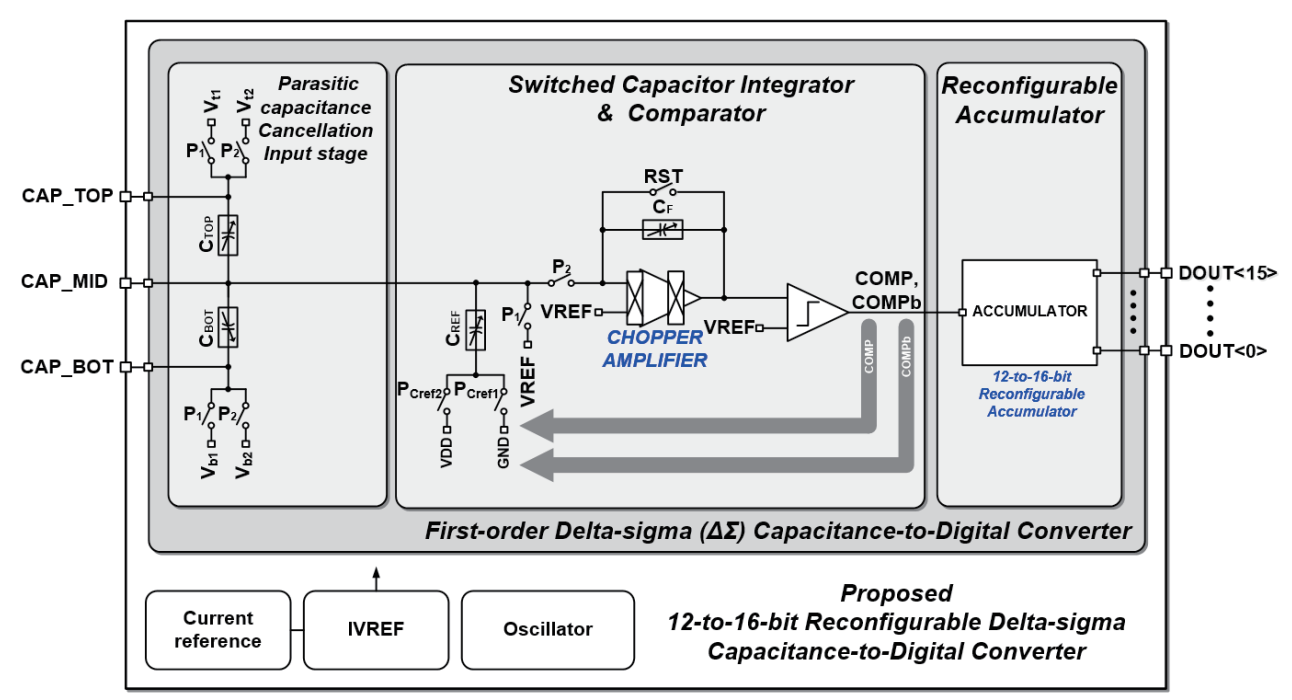

Fig. 1. (Color online) Architecture of the proposed first-order $\Delta \Sigma \mathrm{CDC}$.

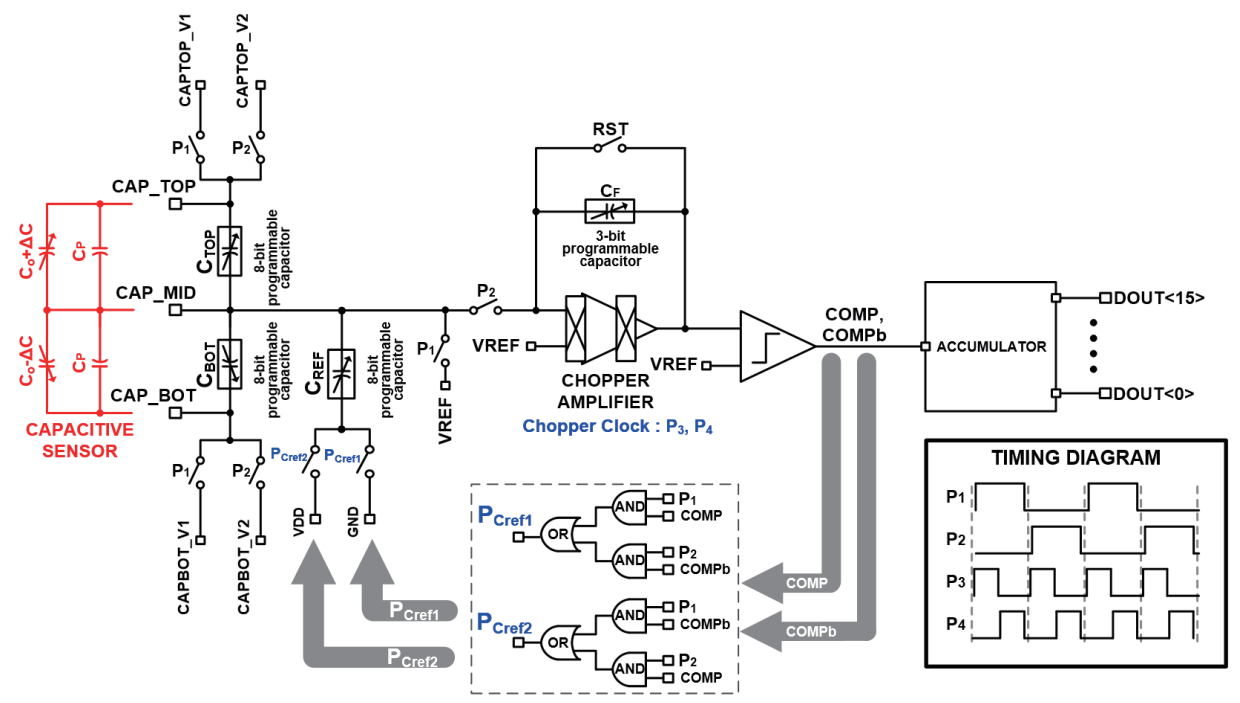

Fig. 2. (Color online) Schematic of the proposed first-order $\Delta \Sigma \mathrm{CDC}$.

integrator output $V_{\text {ref. }}$. The polarity of the charge to be stored in $C_{r e f}$ is then determined on the basis of the output of the comparator. The capacitor sensors operate using a voltage driving method. The driving voltage can use internal voltage VDD and GND, or external arbitrary voltages Cap_top_v1 $\left(V_{t 1}\right)$, Cap_top_v2 $\left(V_{t 2}\right)$, Cap_bot_v1 $\left(V_{b 1}\right)$, and Cap_bot_v1 $\left(V_{b 2}\right)$ are used.

Equation (1) is the result of the charge equation when switching from $\mathrm{P}_{1}$ to $\mathrm{P}_{2}$. A positive polarity is applied when the comparator output is high, and a negative polarity is applied when the comparator output is low. 


$$
\Delta V=\frac{\left(V_{t 1}-V_{t 2}\right)\left(C_{o}+\Delta C\right)+\left(V_{b 1}-V_{b 2}\right)\left(C_{o}-\Delta C\right)+2 C O M P V_{r e f} C_{r e f}}{C_{f}}
$$

The input capacitance range can be obtained using Eqs. (2) and (3). When $\triangle V_{O U T}$ and COMP are low, the minimum $\Delta C$ is obtained.

$$
\Delta C>\frac{\left\{-\left(V_{t 1}-V_{t 2}\right)+\left(V_{b 1}-V_{b 2}\right)\right\} C_{o}-2 V_{r e f} C_{r e f}}{\left(V_{t 1}-V_{t 2}\right)-\left(V_{b 1}-V_{b 2}\right)}
$$

When $\triangle V O<0$ and $C O M P$ remains high, the maximum $\triangle C$ is obtained.

$$
\Delta C<\frac{\left\{-\left(V_{t 1}-V_{t 2}\right)+\left(V_{b 1}-V_{b 2}\right)\right\} C_{o}+2 V_{r e f} C_{r e f}}{\left(V_{t 1}-V_{t 2}\right)-\left(V_{b 1}-V_{b 2}\right)}
$$

The driving voltages $V_{t 1}, V_{t 2}, V_{b 1}$, and $V_{b 2}$ are used to adjust the input range and offset capacitance. Using internal voltage VDD and GND, the input range is given by

$$
-\frac{C_{r e f}}{2}<\Delta C<\frac{C_{r e f}}{2}
$$

where $C_{r e f}$ is an 8-bit programmable capacitor of $100 \mathrm{fF}$ unit capacitance. As such, $C_{r e f}$ has a maximum range of $25.6 \mathrm{pF}$.

The proposed accumulator has dual 16-bit up-counters that count the comparator output and clock $\mathrm{P}_{1}$ (Fig. 3). The first 16-bit up-counter counts the 12 or 16 bits of clock $\mathrm{P}_{1}$. This is

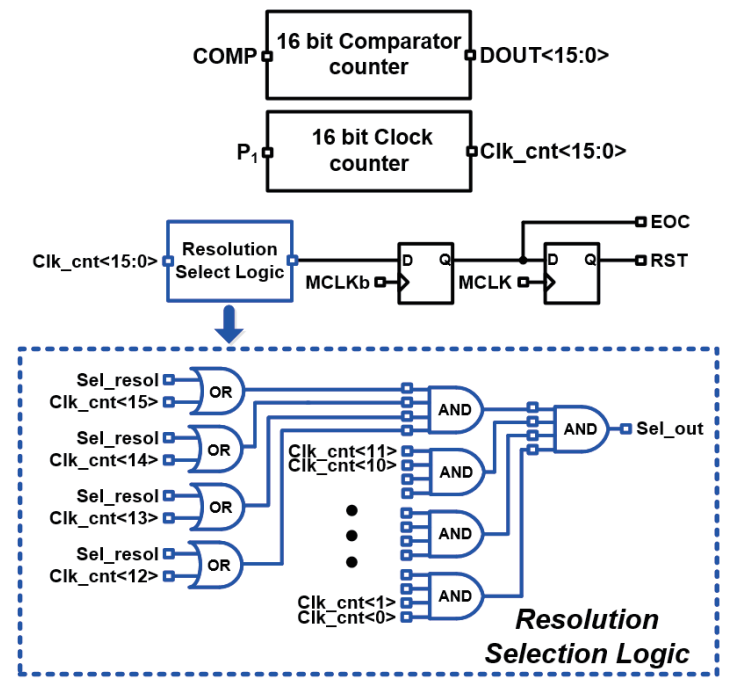

Fig. 3. (Color online) 12- to 16-bit reconfigurable accumulator with dual 16-bit up-counter. 
according to the resolution selection and outputs end-of-clock (EOC) and reset (RST) signals. The EOC and RST signals are output when all 16 outputs of the clock counter are high. If the 12-bit resolution is selected, bits 13 to 16 are set high. The second 16-bit up-counter counts the comparator output until it is reset.

To obtain low noise characteristics, the chopper stabilization technique was applied to the SC $\Delta \Sigma$ modulator. The integrator is of the single-ended folded cascade amplifier type. The chopper was applied inside the integrator. The chopper frequency is twice the sampling frequency. The integrator $1 / f$ noises of the inband are modulated to the outband.

\subsection{Modeling of $\Delta \Sigma$ modulator using MATLAB Simulink}

The effect of chopper stabilization is shown through simulation results using MATLAB Simulink. Figure 4 shows the structure of the MATLAB model of the $\Delta \Sigma$ modulator. The MATLAB model of the $\Delta \Sigma$ modulator consists of a signal generator, an integrator, a 1-bit quantizer, a zero-order hold, a decimation filter, and band-limited white noise.

\section{Design and Simulation Results of Proposed IC}

\subsection{Design of proposed first-order $\Delta \Sigma \mathrm{CDC}$}

The layout of the proposed first-order $\Delta \Sigma \mathrm{CDC}$ is shown in Fig. 5. The $\Delta \Sigma \mathrm{CDC}$ was designed by using the standard $0.18 \mu \mathrm{m} 1 \mathrm{P} 6 \mathrm{M}$ complementary metal-oxide-semiconductor (CMOS) process with an active area of $0.66 \mathrm{~mm}^{2}$. The total current consumption for the 16-bit $\Delta \Sigma \mathrm{CDC}$ is $141 \mu \mathrm{A}$ with a $1.8 \mathrm{~V}$ supply. The current consumption of the analog parts is $68 \mu \mathrm{A}$.

\subsection{Operation of proposed first-order $\Delta \Sigma \mathrm{CDC}$ with chopper stabilization}

The proposed $\Delta \Sigma \mathrm{CDC}$ was simulated using Spectre. The capacitive sensor was modeled as a voltage-controlled variable capacitor for simulation. The input signal is a $1 \mathrm{kHz}$ sine wave, and $\Delta C$ sweeps the minimun-maximum range of $25.6 \mathrm{pF}$. Figure 6 (a) shows the change in capacitance with time. Figure 6(b) shows that the voltage-controlled capacitance and the number of the comparator output "high" is proportional to $\Delta C$.

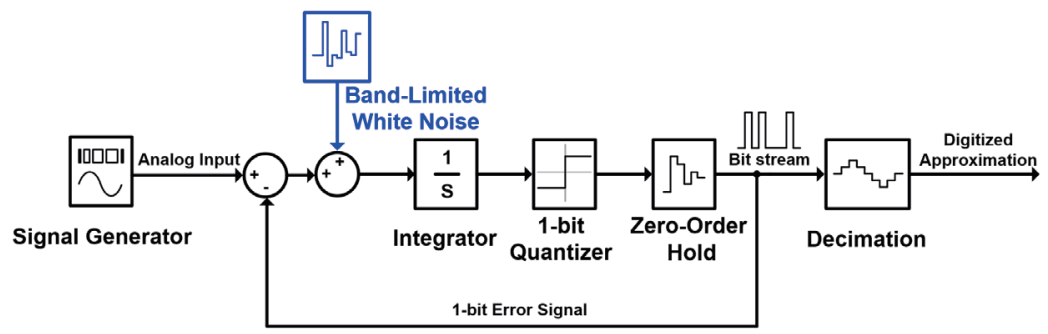

Fig. 4. (Color online) Structure of the MATLAB model of the $\Delta \Sigma$ modulator. 


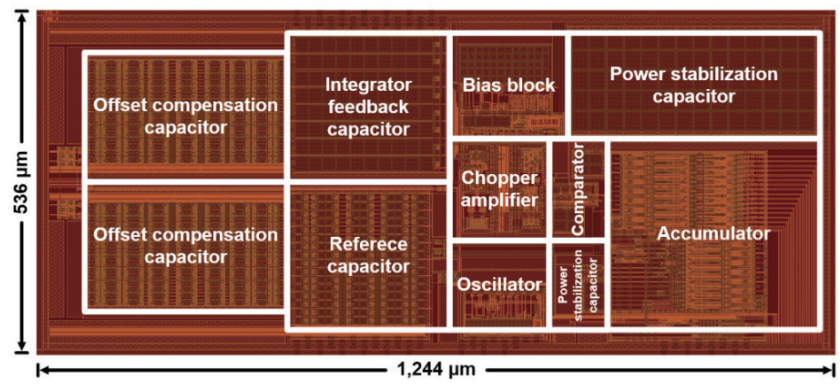

Fig. 5. (Color online) Layout of proposed first-order $\Delta \Sigma \mathrm{CDC}$.

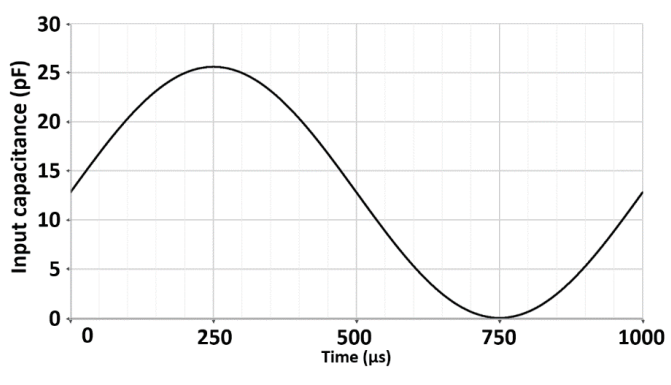

(a)

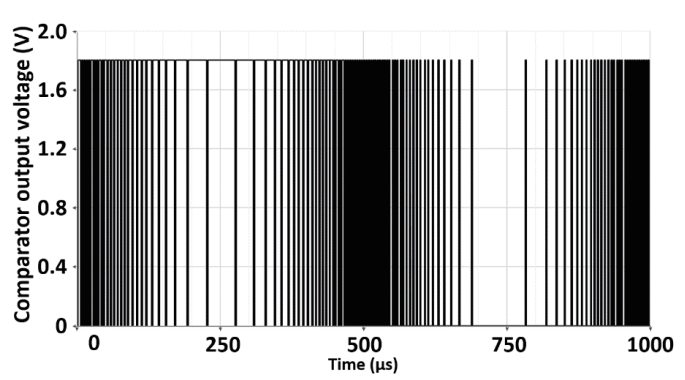

(b)

Fig. 6. (a) Change in capacitance with time and (b) comparator output of the proposed $\Delta \Sigma \mathrm{CDC}$.

Figure 7 shows the input-referred noise of the integrator caused by chopper on/off. The effect of chopper stabilization reduces the input-referred $1 / f$ noise of the SC integrator. The integrated input-referred noises from DC to $100 \mathrm{kHz}$ of chopper on/off are 35 and $147 \mathrm{nV} / \sqrt{\mathrm{Hz}}$.

The fast Fourier transform (FFT) result of the zero-order hold output is shown in Fig 8. The input signal is a $3.1 \mathrm{~Hz}$ sine wave. The input-referred noise results of the proposed integrator are used for MATLAB simulation. In the FFT result of simulation, the signal-to-noise and distortion ratio (SINAD) as well as the effective number of bits $(E N O B)$ are calculated in Eqs. (5) and (6), respectively.

$$
\begin{gathered}
\text { SINAD }=20 \log 10\left(\frac{\text { signal }}{\text { nad }}\right) \\
E N O B=\frac{\text { SINAD }-1.76}{6.02}
\end{gathered}
$$

When calculating SINAD, the chopper improved from 72.72 to $85.18 \mathrm{~dB}$. The $E N O B$ of the proposed $\triangle \Sigma \mathrm{CDC}$ is calculated as 13.8 bits. Without chopper stabilization, the ENOB is 11.8 bits.

A performance summary of parameters and comparisons are noted in Table 1. The proposed first-order $\triangle \Sigma$ CDC achieved an $E N O B$ of 13.8 bits with low noise characteristics. The accumulator for digital code outputs consumes some power but effectively reduces the back-end computing power. 


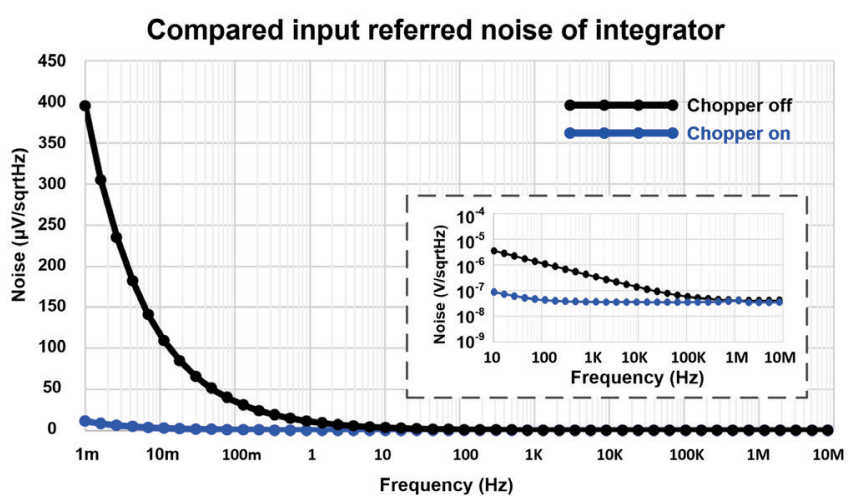

Fig. 7. (Color online) Compared input-referred noise of integrator caused by chopper on/off.

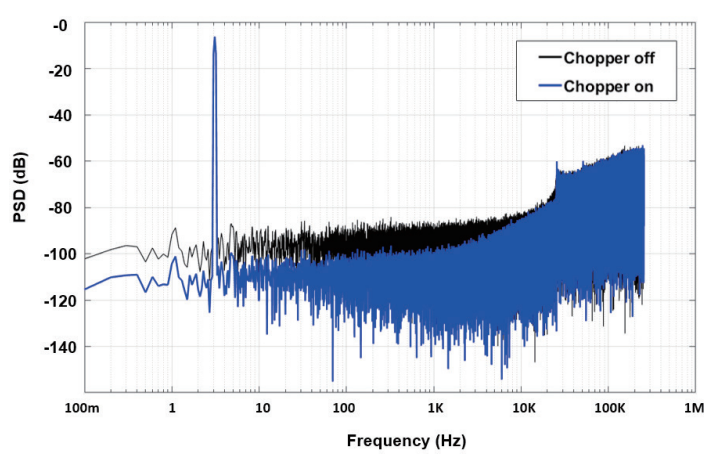

Fig. 8. (Color online) FFT result of comparator output.

Table 1

Performance comparison and summary of parameters.

\begin{tabular}{lccccc}
\hline & This work & Ref. 5 & Ref. 6 & Ref. 7 & Ref. 8 \\
\hline Architecture & 1 st $\Delta \Sigma$ & 3rd $\Delta \Sigma$ & 1 st $\Delta \Sigma$ & 3rd $\Delta \Sigma$ & 3rd $\Delta \Sigma$ \\
Output format & Digital code & Bit stream & Bit stream & Bit stream & Bit stream \\
Technology $(\mu \mathrm{m})$ & 0.18 & 0.35 & 0.35 & 0.16 & 0.35 \\
Supply $(\mathrm{V})$ & 1.8 & 3.3 & 3.3 & $1.2-1.8$ & $1.8-2.5$ \\
Power $(\mu \mathrm{W})$ & 252 & 15000 & 1440 & 10.3 & $828 @ 1.8 \mathrm{~V}$ \\
Active area $\left(\mathrm{mm}^{2}\right)$ & 0.66 & 5.58 & 0.048 & 0.28 & 0.391 \\
Capacitance range $(\mathrm{pF})$ & 25.6 & 10 & $-0.5-0.5$ & $0.54-1.06$ & - \\
ENOB (bit) & 13.8 & 17.2 & 10.2 & 12.5 & 12.2
\end{tabular}

\section{Conclusions}

The low-noise first-order $\Delta \Sigma \mathrm{CDC}$ is presented for the capacitive sensor. The proposed $\Delta \Sigma$ $\mathrm{CDC}$ reduces in-band white noise with noise shaping characteristic as well as low-frequency colored noise with chopper stabilization. Through simulation results, the ENOB of the proposed $\Delta \Sigma$ CDC is 13.8 bits. The chopper stabilization has improved the resolution by 2 bits. The proposed first-order CDC directly converts the capacitance to reconfigurable 12- to 16-bit digital codes and outputs them. To reduce the computing power required for back-end digital processing, the proposed interface circuit fully integrates the 12- to 16-bit reconfigurable accumulator. The layout of the proposed interface circuit occupies an area of $0.66 \mathrm{~mm}^{2}$ in a 0.18 $\mu \mathrm{m}$ 1P6M CMOS process. The total current consumption is $141 \mu \mathrm{A}$ with a $1.8 \mathrm{~V}$ supply.

\section{Acknowledgments}

This work was supported by LeoLSI Co., Ltd. 


\section{References}

1 W. Bracke, P. Merken, R. Puers, and C. V. Hoof: IEEE Trans. Circuits Syst. I Regul. Pap. 54 (2007) 130.

2 K. Tanaka, Y. Kuramochi, T. Kurashina, K. Okada, and A. Matsuzawa: Proc. ASSCC (IEEE, Jeju, Korea, 2007) 244-247.

3 S. Wang and C Dehollain: Proc. IEEE Int. Conf. Electron. Circuits Syst. (ICECS) (2013) 453-456.

4 J. O’Dowd, A. Callanan, G. Banarie, and E. Company-Bosch: Proc. IEEE Sensors (2005) 951-954.

5 S. Xia, K. Makinwa, and S. Nihtianov: IEEE Int. Solid-State Circuits Conf. (ISSCC) Dig. Tech. Papers (2012) 198-200.

6 D. Y. Shin, H. Lee, and S. Kim: IEEE Trans. Circuits Syst. II Express Briefs 58 (2011) 90.

7 Z. Tan, Y. Chae, R. Daamen, A. Humbert, Y. V. Ponomarev, and M. A. P. Pertijs: Symp. VLSI Circuits Dig. Tech. Paper. (2012) 24-25.

8 S. A. Jawed, D. Cattin, M. Gottardi, N. Massari, A. Baschirotto, and A. Simoni: Proc. ESSCIRC (IEEE, Edinburgh, UK, 2008) 442-445.

9 S. Pavan: IEEE Trans. Circuits Syst. I Regul. Pap. 64 (2017) 1953.

\section{About the Authors}

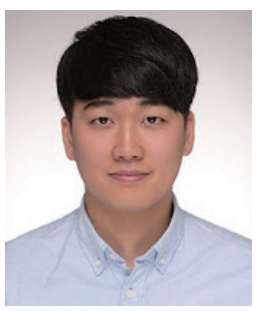

Byeoncheol Lee received his B.S. degree in Electronics Engineering from Chungnam National University, Daejeon, Republic of Korea, in 2017, where he is currently pursuing his M.S. degree. His current research interests are the design of CMOS analog and mixed-mode integrated circuits.

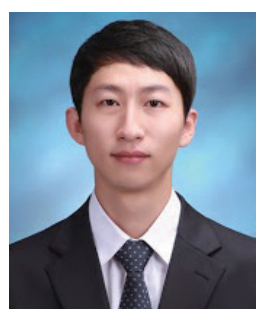

Youngwoon Ko received his B.S. degree in Electronics Engineering from Chungnam National University, Daejeon, Republic of Korea, in 2015, where he is currently pursuing his M.S. degree. His current research interests are the design of CMOS analog and mixed-mode integrated circuits.

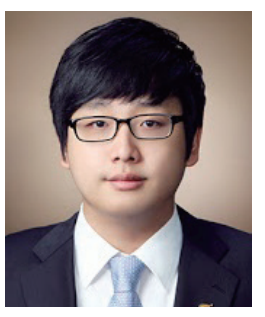

Hyungseup Kim received his B.S. degree in Electronics Engineering from Chungnam National University, Daejeon, Republic of Korea, in 2014, where he is currently pursuing his Ph.D. degree. His current research interests are the design of CMOS analog and mixed-mode integrated circuits. 


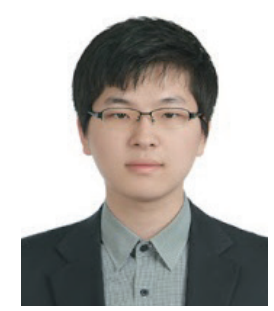

Yeongjin Mun received his B.S. degree in Electronics Engineering from Chungnam National University, Daejeon, Republic of Korea, in 2016, where he is currently pursuing his M.S. degree. His current research interests are the design of CMOS analog and mixed-mode integrated circuits.

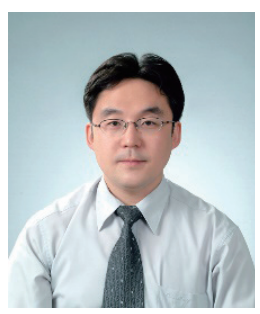

Seounghoon Huh worked with Samsung Electronics as a Marketing Manager from 1988. In 2007, he founded LeoLSI, where he has been working as the CEO.

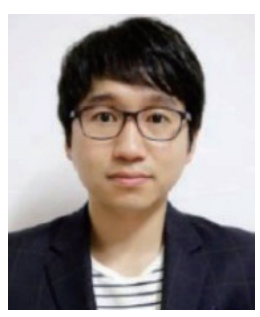

Dongkyu Song received his B.S. and M.S. degrees in Computer Engineering from Seokyeong University, Republic of Korea, in 2007 and 2009, respectively. In 2009, he joined LeoLSI Co., Ltd., Republic of Korea, where he is now a senior engineer. His current research interests are the design of CMOS analog integrated circuits, CMOS mixed-mode integrated circuits, and capacitive sensor readout circuitry.

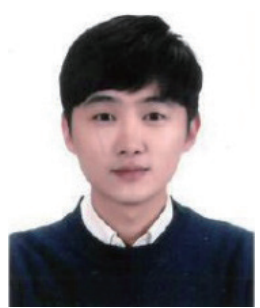

Youngtaek Roh received his B.S. degree in Electronics Engineering from Seokyeong University, Seoul, Republic of Korea, in 2013. In 2012, he joined LeoLSI Co., Ltd., Republic of Korea, where he is now an associate research engineer. His current research interests are the design of CMOS analog integrated circuits, CMOS mixed-mode integrated circuits, and capacitive sensor readout circuitry.

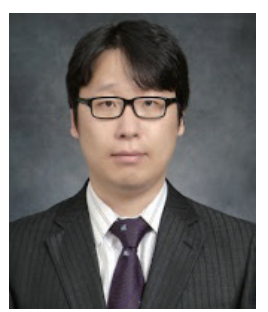

Hyoungho Ko received his B.S. and Ph.D. degrees in Electrical Engineering from Seoul National University, Republic of Korea, in 2003 and 2008, respectively. From 2008 to 2010, he worked with Samsung Electronics as a senior engineer. In 2010, he joined the Department of Electronics Engineering at Chungnam National University, Republic of Korea, where he is currently an associate professor. His current research interests are the design of CMOS analog and mixed-mode integrated circuits. 